\title{
Transient equilibrium mapping: A new tool for measuring the thermodynamics of slowly assembling supramolecular systems
}

Christopher D. Hennecker, ${ }^{1}$ Christophe Lachance-Brais, ${ }^{1}$ Hanadi Sleiman, ${ }^{1}$ and Anthony Mittermaier ${ }^{1,{ }^{*}}$

${ }^{1}$ Department of Chemistry, McGill University. 801 Sherbrooke St. W. Montreal, Canada H3A OB8

*To whom correspondence should be addressed: anthony.mittermaier@mcgill.ca

Supramolecular chemistry involves the non-covalent assembly of monomers into materials with unique properties and wide-ranging applications. Thermal analysis is one of the key analytical tools in this field, as it provides quantitative thermodynamic information on both the structural stability and nature of the underlying molecular interactions. However there exist many supramolecular systems whose kinetics are so slow under conditions approaching chemical equilibrium that thermodynamic data are inaccessible. We have developed a simple, general, and rapid spectroscopic method for extracting thermodynamic parameters from such slowly assembling systems. It is based on repeatedly raising and lowering the temperature during assembly and identifying the points of transient equilibrium as they are passed on the up- and down-scans. In a proof-of-principle application to the slow co-assembly of polydeoxyadenosine containing 15 adenosines (poly $\mathrm{A}_{15}$ ) and cyanuric acid (CA), we found that roughly $30 \%$ of the CA binding sites on the polyA chains were unoccupied, with implications for the assembly of high-valence systems. 


\section{Introduction}

Supramolecular chemistry is emerging as a rich source of diverse materials with novel and valuable properties. Potential applications range from drug-delivery and tissue regeneration to optical sensors and organic electronics. ${ }^{1}$ This approach involves the non-covalent self-assembly of tens to thousands of monomeric units into larger structures with emergent physical properties that derive from both the structures of the individual components and their interactions and arrangement with respect to one another. ${ }^{2}$ Reversible assembly has some distinct advantages compared to traditional covalent synthesis. The dynamic nature of supramolecular interactions allows bonds to break and reform leading to materials with self-healing properties. Furthermore, many supramolecular systems have the ability to generate multiple morphologies and sets of physical properties from a single set of building blocks with only small modifications of the assembly conditions. ${ }^{3}$ Nevertheless, there are unique challenges associated with this approach. Chief among these is characterizing the products of a non-covalent assembly reaction. Much of the excitement surrounding supramolecular chemistry comes from the fact that there remains much to be understood regarding the relationships between the chemical structures of the monomeric units, the supramolecular architectures, and the emerging physical properties, and there is wide possibility for new and unexpected discoveries. However, this implies that the nature of supramolecular products is difficult to predict and that rigorous structural and thermodynamic analyses are critical to advancing the field.

A variety of tools have been used to elucidate the structures produced by assembly, including atomic force, electron, and super-resolution microscopies, and solid-state NMR spectroscopy. ${ }^{4-6}$ The stabilities of the assemblies are most commonly measured by thermal analysis. Most supramolecular structures dissociate when they are heated and reassemble when the monomer mixtures are cooled. This process can be quantified either by calorimetry ${ }^{7}$ or by spectroscopically-detected melting and annealing. ${ }^{8}$ Detailed analyses of melting curves provide substantial insight into the forces holding together the supramolecular structures and yield additional thermodynamic parameters such as the enthalpies, $\Delta H$, entropies $\Delta S$, and free energies, $\Delta G$, of critical steps in the assembly process. ${ }^{9}$ However, there exists a subset of supramolecular systems for which thermal analysis fails to give reliable thermodynamic data due to extremely slow assembly kinetics. Such systems only assemble or disassemble at useful rates when they are pushed far from equilibrium, i.e. under very highly stabilizing or destabilizing conditions. Common examples include amyloid fibrils, viral capsids, and a variety of self-assembling non-biological small molecules. ${ }^{10-16}$ Thermodynamic analyses rely on assembled and disassembled monomers existing at their equilibrium distributions over the range of temperature measured during the experiment. Slowly assembling systems require so much time to equilibrate that this is not achievable on practical timeframes using standard melting and annealing experiments. For this reason, rigorous thermodynamic information is largely missing for slowly assembling systems, hampering progress and discouraging their study.

We have developed a new experimental approach we term transient equilibrium (TE) mapping that gives rigorous thermodynamic data on slowly assembling systems and can be performed using a standard temperature-controlled spectrophotometer. Rather than waiting for the system to equilibrate (which can take days or weeks), the temperature is repeatedly raised and lowered, driving cyclic, non-equilibrium disassembly and assembly. Since the $T_{m}$ lies between the starting and ending temperatures of the scans, 
the system briefly passes through a moment of transient equilibrium on each up-scan and downscan. The transient equilibria (temperature and concentration values) can be clearly identified from the spectroscopic trace, allowing the full equilibrium melting curve to be mapped out in just a few hours.

For example, we applied TE mapping to better understand the recently discovered co-assembly of polydeoxyadenosine (polyA) and the small molecule cyanuric acid (CA) into fibres whose biocompatibility and low cost make them promising candidates for tissue engineering and drug delivery. ${ }^{17} \mathrm{~A}$ crosssection of the proposed structure (Figure 1) shows the adenosine of three different DNA strands hydrogen bonding to CA molecules in a continuous supramolecular helicene. ${ }^{18}$ We note that the ideal helicene structure has a 1:1 ratio of $d A$ residues and CA molecules. We recently characterized the kinetics of polyA-CA fibre assembly using nonequilibrium melting methods. ${ }^{14}$ Equilibration of the fibres near the melting point could take up to a month of constant instrument use. Using TE mapping, we determined the $\Delta G, \Delta H$, and $\Delta S$ values for adding a polyA chain to the end of a growing fibre in a single 10-hour experiment. By repeating these measurements at different concentrations of $\mathrm{CA}$, we determined the minimum polyA:CA ratio necessary for assembly and made the surprising
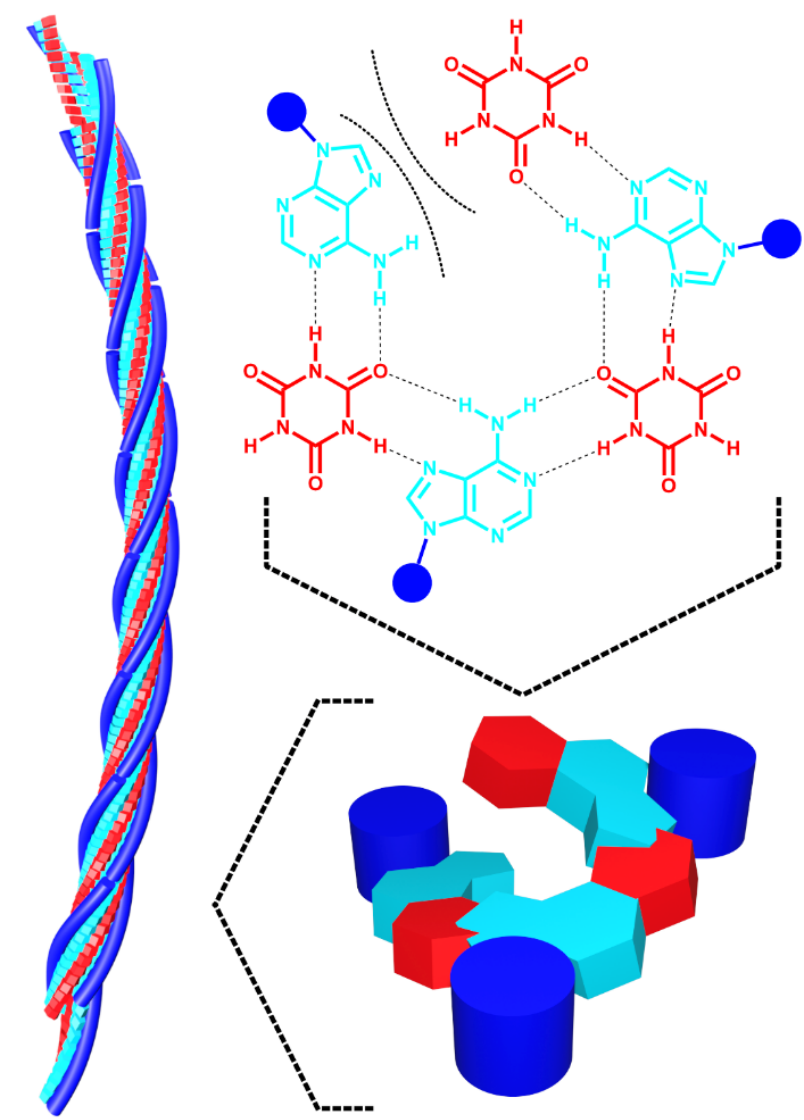

Figure 1: Proposed structure of supramolecular fibers formed from the co-assembly of polyadenosine strands and cyanuric acid (left). A cross section of a single hexameric helicene (right). discovery that about $30 \%$ of the available CA binding sites are unfilled under our conditions. These results have implications for the future development of asymmetric systems involving components of very different valences, such as polyA and CA, and demonstrate the potential of the TE approach for learning about slowly assembling systems.

\section{Results and Discussion}

\section{Theory}

We previously characterized the kinetics of polyA-CA fibre assembly using thermal hysteresis melting and annealing experiments in which the temperature is varied and the concentration of free monomers is detected spectroscopically. For slowly assembling systems, such as polyA-CA, the rate at which the system relaxes to equilibrium is far slower than the temperature scan rate, thus both folding (cooling) and unfolding (heating) occur out of equilibrium. The populations effectively lag behind the changing temperature such that the cooling and heating scans are offset, with the apparent $T_{m}$ reached at a higher temperature than the true $T_{m}$ on the up-scan and at a lower temperature than the true $T_{m}$ on the downscan, as illustrated in Figure $2 a$. The resulting hysteresis loops can be analyzed to give folding and 
unfolding rates and activation energies in the case of 2-state folding reactions. ${ }^{9}$ For fibres, as is the case here, hysteresis data can be fit to more complicated assembly schemes, ${ }^{14,17}$ such as the Goldstein-Stryer (GS) cooperative kinetic model, where $\mathrm{M}_{\mathrm{N}}$ describes a fibre with $N$ polyA chains: ${ }^{19}$

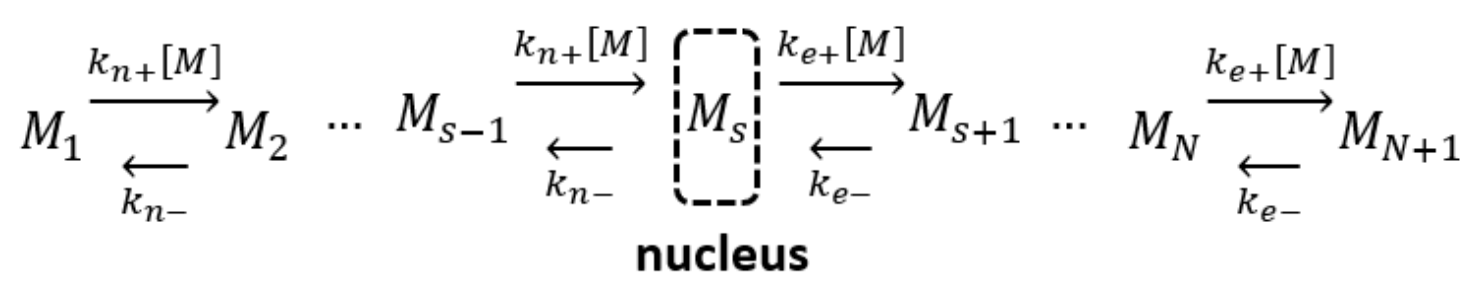

Association and dissociation of polyA monomers (i.e. $\mathrm{dA}_{15}$ oligodeoxynucleotides, $M_{1}$ ) and short oligomers less than the critical nucleus size, $s$, are described by the nucleation rate constants $k_{n+}$ and $k_{n-}$ respectively, while oligomers larger than $s$ are described with the elongation rate constants $k_{e^{+}}$and $k_{e_{-}}$. All four rate constants are typically assumed to obey Arrhenius kinetics with activation energies $\mathrm{E}_{n+}{ }^{\mathrm{a}}, \mathrm{E}_{\mathrm{n}-}{ }^{\mathrm{a}}, \mathrm{E}_{\mathrm{e}+}{ }^{\mathrm{a}}$, and $\mathrm{E}_{\mathrm{e}-}$ a. Fits to the hysteresis data give the four rate constants and four activation enthalpies. In principle, these values contain thermodynamic information. For example, the ratio $k_{e-} / k_{e+}=\mathrm{K}_{\mathrm{e}}$ is the equilibrium dissociation constant for adding a polyA chain to the end of an elongating fibre. The differences $\Delta \mathrm{H}_{\mathrm{e}}=\mathrm{E}_{\mathrm{e}}$ ${ }^{a}-E_{e+}{ }^{a}$ and $\Delta S_{e}=\Delta H_{e} / T+R \ln \left(K_{e}\right)$ are the enthalpy and entropy changes for the same process, respectively. In practice, however, thermodynamic parameters are very poorly defined by the hysteresis data. To illustrate, simulated GS fibres with equilibrium melting temperatures spanning over 10 degrees can produce virtually superimposable hysteresis data (Figure 2a). Similarly, experimental hysteresis data for polyA-CA fibres give visually very similar fits, even when $\Delta H_{e}$ and $\Delta S_{e}$ values are varied by a factor of 1.6
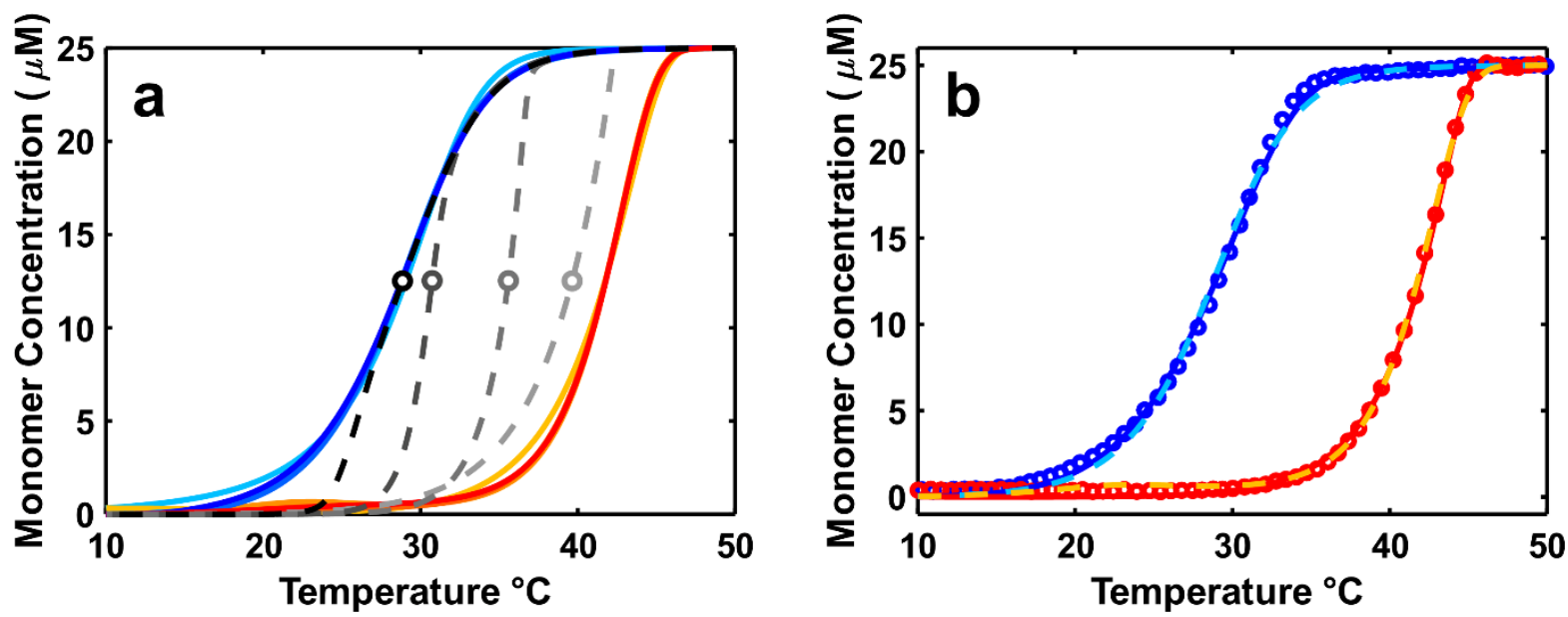

Figure 2: a) Fiber assembly/disassembly simulated using the Goldstein-Stryer model and kinetic parameters that give similar melting and annealing curves (solid lines) with drastically different equilibrium curves (dashed lines). Heating curves are shown in red/orange and cooling curves are shown in blue/cyan. The offset between heating and cooling data is due to thermal hysteresis (TH). Simulation parameters are listed in Supplementary Table 1 b) Fits of experimental $\mathrm{TH}$ curves with $\Delta \mathrm{H}_{e}$ extracted from $\mathrm{TH}$ data alone (blue and red solid lines) and $\Delta H_{e}$ constrained to be equal to the value extracted from TE measurements (dashed cyan and orange lines). Simulation parameters are listed in Supplementary Table 2 
(Figure $2 b$, Supplementary Table 1). Thus new approaches are needed to accurately determine thermodynamic parameters for slowly-equilibrating systems.

Recent work from the Yamaguchi $\mathrm{lab}^{16}$ has explored how the spectra of slowly equilibrating, self assembling systems respond to repeated heating and cooling cycles. ${ }^{20}$ Depending on the starting and ending temperatures and ramp rates, a rich diversity of shapes (thermal hysteresis loops) have been observed, providing qualitative information on the underlying assembly reactions. However, to date there has not been a straightforward way to extract quantitative thermodynamic information from these data. Our new TE mapping approach uniquely fills this gap. In order to illustrate the fundamental principles, we performed kinetic simulations using the GS assembly model and parameters for polyA-CA fibres (Figure $3 a$, see Supplementary Information). The dashed black line indicates the concentration of monomers that would be present after extensive equilibration, i.e. the critical monomer concentration, $[\mathrm{M}]_{\mathrm{c}}$, as a function of temperature. This curve is rich in thermodynamic information and can be analyzed to obtain $\Delta \mathrm{H}_{\mathrm{e}}, \Delta \mathrm{S}_{\mathrm{e}}$,
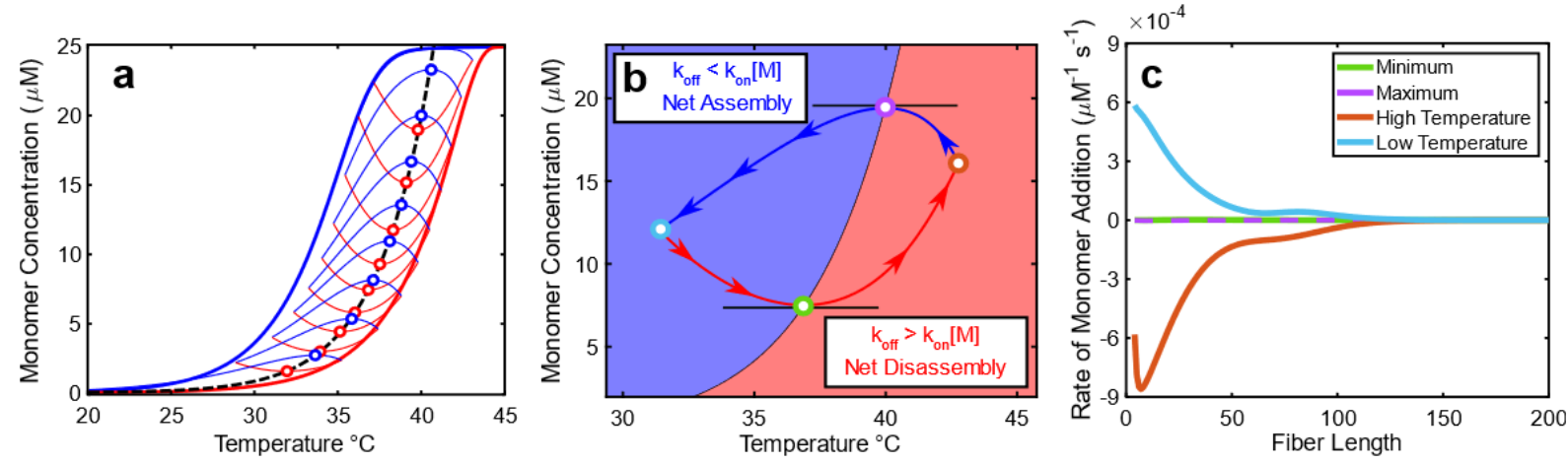

Figure 3: a) Kinetic simulations of a typical hysteresis experiment (bold lines) and TE mapping experiment (narrow lines). Cooling traces are shown in blue, heating traces are shown in red. The experiment begins by cooling from $45^{\circ} \mathrm{C}$ to $36^{\circ} \mathrm{C}$, this is followed by the first up-scan $\left(36^{\circ} \mathrm{C}\right.$ to $\left.44^{\circ} \mathrm{C}\right)$, a second down-scan $\left(44^{\circ} \mathrm{C}\right.$ to $\left.35^{\circ} \mathrm{C}\right)$, a second up-scan $\left(35^{\circ} \mathrm{C}\right.$ to $\left.43^{\circ} \mathrm{C}\right)$, a third down-scan $\left(43^{\circ} \mathrm{C}\right.$ to $\left.34^{\circ} \mathrm{C}\right)$, etc. The equilibrium profile is shown as the dashed black line, with the extrema of each TE cycle shown as points. b) An isolated TE mapping cycle: assembly occurs only in the blue shaded region; disassembly only occurs in the red shaded region. The interface of these two regions represents a system at equilibrium. Coloured points represent the position of calculated monomer flux in panel c. c) Calculated monomer flux of fibers for points shown in panel $b$, the horizontal extrema of the TE cycle have 100-fold less flux then either the high or low temperature values.

and $\mathrm{K}_{\mathrm{e}}$, as well as the corresponding parameters for fibre nucleation. ${ }^{21}$ For a rapidly-equilibrating system, the $[\mathrm{M}]_{\mathrm{c}}$ curve can be measured directly by traditional melting or reannealing experiments. ${ }^{21}$ In contrast, for the slowly assembling polyA-CA system, heating and cooling scans give the left- and right-shifted blue and red curves, respectively, and the location of the $[\mathrm{M}]_{\mathrm{c}}$ curve is obscured by the thermal hysteresis, as illustrated in Figure $3 a$.

The TE mapping method is based on our discovery that repeatedly raising and lowering the temperature such that it repeatedly traverses the true $T_{m}$ reveals the precise locations of the hidden equilibria. Simulating TE data for polyA-CA assembly gives a series of concave-up and concave-down arcs on the heating and cooling scans, respectively (narrow red and blue curves in Figure $3 a$ ). Strikingly, the $[\mathrm{M}]_{\mathrm{c}}$ values (black line) pass directly through the extrema (concentration maxima and minima) of the cooling and heating arcs. Thus experimentally-determined extrema can be interpreted as a set of $[M]_{c}(T)$ values. 
The physical process underlying this behaviour can be understood as follows: for cooperatively assembled fibres, such as polyA-CA, equilibrium is reached when the rate of monomer addition to the end of a fibre $\left(k_{e+}[M]_{c}\right)$ is exactly equal to the rate of monomer dissociation from the end of a fibre $\left(k_{e}-\right)$, such that the net rate of fibre growth is zero. ${ }^{21}$ When $\left[M_{1}\right]<[M]_{c}$ there is net dissociation and $\left[M_{1}\right]$ increases with time, corresponding to the red region below the $[\mathrm{M}]_{\mathrm{c}}$ curve in Figure $3 b$. When $\left[\mathrm{M}_{1}\right]>[\mathrm{M}]_{\mathrm{c}}$ there is net association and $\left[M_{1}\right]$ decreases with time, corresponding to the blue region above the $[M]_{c}$ curve. Every cooling scan starts in the red region with net dissociation (increasing $\left[\mathrm{M}_{1}\right]$ ) and ends in the blue region with net association (decreasing $\left[M_{1}\right]$ ). As the temperature crosses the boundary where $\left[M_{1}\right]=[M]_{c}$, net fibre growth is zero, the arc is exactly horizontal, and the maximum is reached. Conversely, every heating scan starts in the blue region with decreasing $\left[\mathrm{M}_{1}\right]$ and ends in the red region with increasing $\left[\mathrm{M}_{1}\right]$. As the temperature crosses the $\left[M_{1}\right]=[M]_{c}$ boundary, the free monomer concentration is at a minimum. To validate this interpretation, we calculated the net rate of monomer addition to each length of fibre in the simulation. At the lower and upper limiting scan temperatures (orange and cyan), the rates of monomer addition and release are at least 100 -fold greater than at the horizontal extrema of the heating and cooling arcs (green and purple) (Figure $3 c$ ). Furthermore, since $\mathrm{k}_{\mathrm{e}+}[\mathrm{M}]_{\mathrm{c}}=\mathrm{k}_{\mathrm{e}-}$ at all of the extrema, this implies that the experimental monomer concentrations at these points are equal to the equilibrium dissociation constant $K_{e}=\mathrm{k}_{\mathrm{e}}-/ \mathrm{k}_{\mathrm{e}+}$. In other words, thermodynamic parameters can be read directly from TE data.
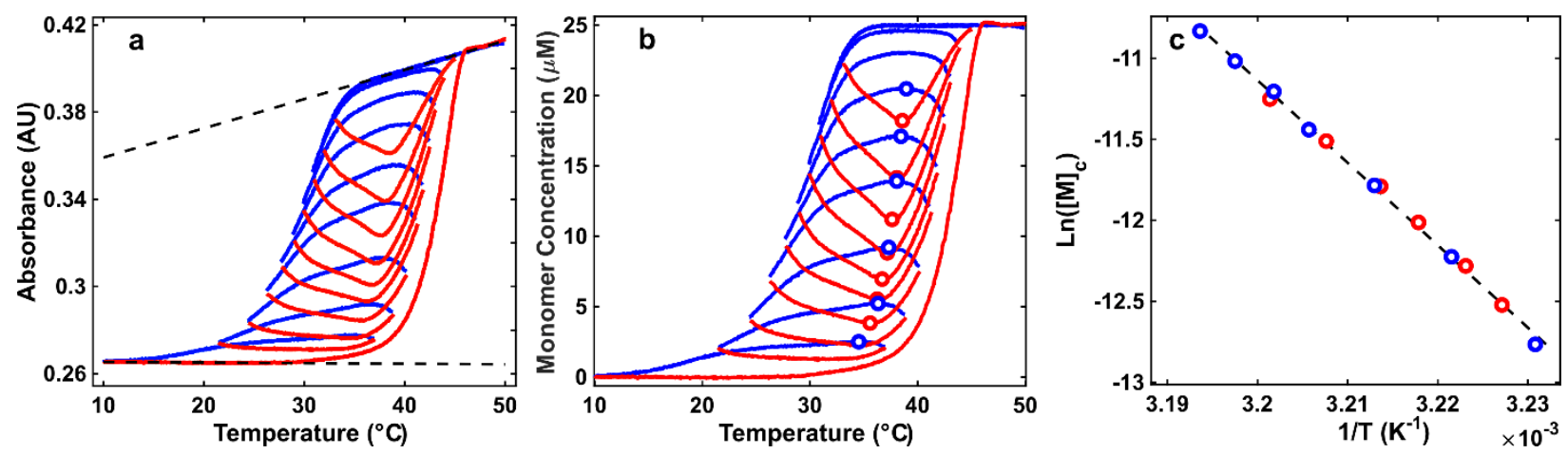

Figure 4: a) Raw absorbance data for a 15mer polyA-CA coassembly with $25 \mu \mathrm{M}$ poly $A_{15}$ and $15 \mathrm{mM} C A$ at $\mathrm{pH} 4.5$, blue lines represent cooling traces and red lines represent heating traces. Unfolded (top black line) and folded (bottom black line) are also shown. b) Processed TE data with extrema of each cycle shown as points. c) Van 't Hoff analysis of experimental TE points, plotted along with confidence interval of one standard deviation.

\section{Analysis of experimental TE data}

We performed a TE experiment on a mixture of CA and polyA chains by repeatedly raising and lowering the temperature over a window of roughly $15^{\circ} \mathrm{C}$ that shifted from (29-44) to (14-33) ${ }^{\circ} \mathrm{C}$ in 9 cycles while we monitored the spectroscopic absorbance at $260 \mathrm{~nm}$ (Figure 4a, see Supplementary Information for a guide to selecting sliding T-windows). The lower and upper absorbance regions were fitted to linear baselines and assigned $100 \%$ and $0 \%$ folded, i.e. $\left[\mathrm{M}_{1}\right]=0$ and $25 \mu \mathrm{M}$, respectively. The converted data are shown in Figure $4 b$, with blue and red indicating cooling and heating, respectively, and open circles placed at the extrema. These experimental arcs have a remarkable similarity to the calculations shown in Figure $3 a$. The $y$ - and $x$-values of the extrema correspond directly to critical monomer concentration, $[\mathrm{M}]_{c}$, and temperature pairs. As discussed above, $[\mathrm{M}]_{\mathrm{c}}$ values are equivalent to the equilibrium dissociation 
constant, $K_{e}$, for adding a polyA to the end of an elongating fibre, for this system. A van 't Hoff plot of $\ln \left([\mathrm{M}]_{\mathrm{c}}\right)=\ln \left(K_{e}\right)$ vs $1 / \mathrm{T}$ is linear with a slope of $-\Delta \mathrm{H}_{\mathrm{e}} / \mathrm{R}$ and $\mathrm{y}$-intercept of $\Delta \mathrm{S}_{\mathrm{e}} / \mathrm{R}$ (Figure $4 c$ ), giving $\Delta \mathrm{H}_{\mathrm{e}}=100$ $\pm 2 \mathrm{kcal} \mathrm{mol}^{-1}$ and $\Delta \mathrm{S}_{\mathrm{e}}=301 \pm 7 \mathrm{cal} \mathrm{mol}^{-1} \mathrm{~K}^{-1}$. Notably, although the values of $\Delta \mathrm{H}_{\mathrm{e}}$ and $\Delta \mathrm{S}_{\mathrm{e}}$ determined by TE mapping differ from those obtained by kinetic fits to TH data by factors of 1.6 (Supplementary Table 2), repeating the TH analysis with $\Delta \mathrm{H}_{\mathrm{e}}$ and $\Delta \mathrm{S}_{\mathrm{e}}$ fixed to the TE-derived values gives good agreement with experimental data (Figure $2 b$ ), illustrating the insensitivity of the kinetic fits to these thermodynamic parameters. In general, we would strongly recommend that, even if assembly kinetics are the main interest, the combination of TE and thermal hysteresis experiments provide more robust solutions than thermal hysteresis alone, as TE resolves ambiguity in the fitted rate constants and ratios thereof.

Furthermore, the thermodynamic parameters provide a basis for comparing polyA-CA fibres to other nucleic acid structures. For example, polyA/polyT $\left(\mathrm{dA}_{15} \mathrm{dT}_{15}\right)$ duplex dissociation is predicted to have approximately $\Delta \mathrm{H}=108 \mathrm{kcal} \mathrm{mol}^{-1}$ and $\Delta \mathrm{S}=335 \mathrm{cal} \mathrm{mol}^{-1} \mathrm{~K}^{-1}$ under similar solution conditions to those used here, ${ }^{22}$ It is intriguingly similar to the values we measured for polyA-CA assembly $\left(100 \mathrm{kcal} \mathrm{mol}^{-1}\right.$ and $301 \mathrm{cal} \mathrm{mol}^{-1} \mathrm{~K}^{-1}$ ). At first glance, we would have expected polyA-CA fibres to show much higher enthalpies and entropies than dAdT duplexes, since there are three strands rather than two, each dA forms twice as many hydrogen bonds and immobilizes a CA molecule in the putative polyA-CA structure (Figure 1). However partial vacancy of CA binding sites may help to reconcile these observations, as elaborated below.

\section{Stoichiometry of polyA-CA fibres.}

One of the great advantages of quantitative thermodynamic data is that much can be learned about the system of interest through careful analyses of how energetic parameters vary with changing conditions. For instance, the presumptive structure of polyA-CA fibres shows that one molecule of CA is present for every deoxyadenosine residue in each polyA chain. In other words, when one of the $\mathrm{dA}_{15}$ polyA chains binds the end of an elongating fibre, it should be accompanied by 15 CA molecules. While equilibrium dialysis experiments are consistent with this structure, ${ }^{17}$ they have relatively low precision and the stoichiometry is very difficult to measure with accuracy. This property is of great interest since a CA:polyA stoichiometry of less than 15 would reveal the existence of defects, which could potentially be targeted with other small molecules. Thermodynamic data can help to resolve this issue, since the apparent dissociation constant, $K_{e}$, for a polyA chain binding to the end of the fibre should vary with CA concentration in a predictable way. For instance, if a polyA chain always brings with it $c$ molecules of CA, i.e.

$$
M_{n}+M_{1}+c C A \stackrel{K_{e q}}{\leftrightarrow} M_{n+1}
$$

(following the nomenclature of Equation 1), then the full equilibrium dissociation constant for the process is given by

$$
K_{(T)}^{\circ}=\frac{\left[M_{n}\right]\left[M_{1}\right][C A]^{c}}{\left[M_{n+1}\right]}
$$

This is something of an over-simplification, as elaborated below, but for now it serves to illustrate the dependence of $K_{e}$ on [CA]. For polyA-CA fibres, CA is always in great excess so that its concentration is effectively constant for any set of assembly conditions. The apparent polyA dissociation constant $K_{e}$ is related to the full equilibrium constant according to 


$$
K_{e}=\left.\frac{\left[M_{n}\right]\left[M_{1}\right]}{\left[M_{n+1}\right]}\right|_{[C A]}=K_{(T)}^{\circ}[C A]^{-c}
$$

with the temperature dependence of the standard equilibrium constant $\left(K_{(T)}^{\circ}\right)$ described by

$$
K_{(T)}^{\circ}=\exp \left(-\frac{\left(\Delta H_{(T)}-T \Delta S_{(T)}\right)}{R T}\right)
$$

Therefore, measuring $K_{e}$ at a series of different CA concentrations should produce offset van 't Hoff plots where the vertical distance between each line follows the stoichiometry of CA. To proceed, we noted that stabilization of polyA-CA fibres at high [CA] is largely entropic in nature, since it is primarily driven by differences in the entropy of dilution when dissociation of a polyA chain concomitantly releases $c$ molecules of CA into solution.

We repeated the TE experiment at four CA concentrations between 7.5 and $15 \mathrm{mM}$ (Supplementary Figure 1). Van 't Hoff plots of the resulting $K_{e}$ values are shown in Figure 5. Fitting Equation 4 to this data set allows us to directly obtain the stoichiometry of CA. To account for the possibility of a temperature dependent enthalpy value we extracted global values of $\Delta \mathrm{H}_{\mathrm{e}}$ and $\Delta \mathrm{C}_{\mathrm{p}}$. The heat capacity change of binding, $\Delta C_{p}$, accounts for any temperature-dependent differences in the slopes of the different experiments according to:

$$
\begin{gathered}
\Delta H_{e}(T)=\Delta H_{e}\left(T_{0}\right)+\Delta C_{p}\left(T-T_{0}\right) \\
\Delta S_{e}(T)=\Delta S_{e}\left(T_{0}\right)+\Delta C_{p} \ln \left(\frac{T}{T_{0}}\right)
\end{gathered}
$$

The extracted $\Delta C_{p}=-0.6 \pm 0.3 \mathrm{kcal} \mathrm{mol}^{-1} \mathrm{~K}^{-1}$ indicates that the enthalpy of adding a polyA chain to a growing fibre has only a slight temperature dependence. This is perhaps unsurprising, since $\Delta C_{p}$ values associated with nucleic acid folding are largely sequence dependent and have been observed to vary from slightly negative to positive values. ${ }^{23}$ The global fit was in good agreement with experimental data points (Figure 5, Supplementary Table 3). Surprisingly, the extracted stoichiometry coefficient, $c=10.4 \pm 0.6$, implies that $30 \%$ of possible CA binding sites are unoccupied in polyA-CA fibres under these conditions.

\section{Master equations for high-valence assembly}

The thermodynamics of multivalent supramolecular assembly can be summarized in terms of two main trends: the "principle of maximum occupancy" which refers to the tendency of systems to evolve toward the most stable state with full occupancy of binding sites, and the "entropy factor" which favours the state of the system with the largest number of product species. ${ }^{24}$ For most of the supramolecular systems studied to date, the valency (number of binding sites per monomer) is relatively small $(<6)$, the principle of maximum occupancy dominates, and the all sites are generally filled in the assembled materials. ${ }^{25} 26$ However, for high-valence monomers, such as the polyA chains studied here, the entropy factor strongly opposes the principle of maximum occupancy and more complex behaviour emerges. For example, each $\mathrm{dA}_{15}$ chain creates an additional 15 potential CA binding sites, on average, as it adds to the end of growing fibre; one site must be created for each additional $d A$ residue to achieve the theoretical 1:1 dA:CA stoichiometry. The number of ways to fill $c$ of the 15 binding sites with $c$ molecules of CA is given by the binomial coefficient ${ }^{27}$ 


$$
N_{c}=\frac{15 !}{c !(15-c) !}
$$

While there is only $N=1$ way completely fill all 15 binding sites $(c=15)$, there exists a total of $N=32,766$ distinct ways fill the sites with $1 \leq c \leq 14$ molecules of CA. A simplified model of this energy diagram is seen in Figure $6 b$, where partially filled states are higher in energy but are more numerous. Therefore, even though a polyA chain with 15 bound CA molecules may represent the single lowest energy configuration, there exists such an enormous number of partly-filled configurations that these dominate, with a broad distribution of CA uptake and just 10 of the 15 sites being filled on average as seen in Figure $6 c$.

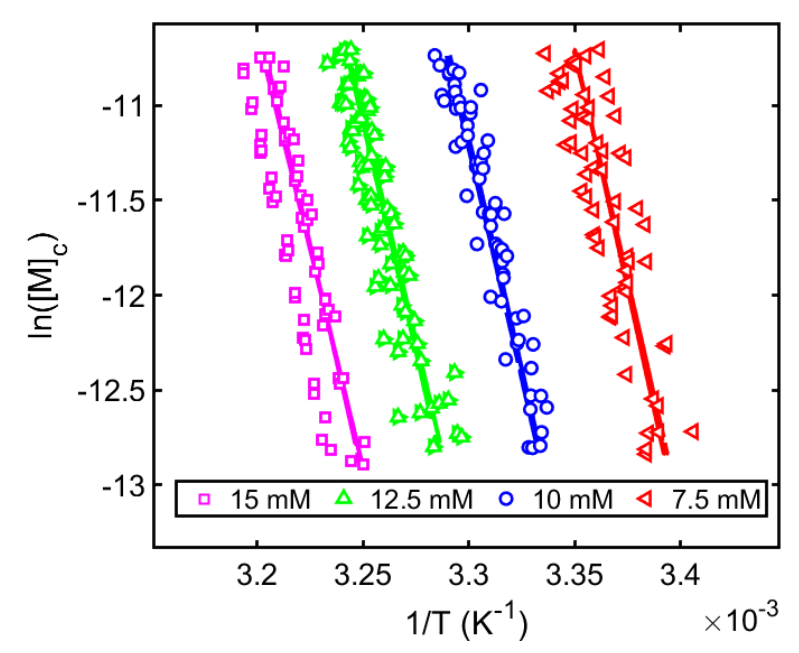

This explanation implies that polyA chains can bring a variable number of $\mathrm{CA}$ molecules with them when they attach to the end of a growing fibre, which is inconsistent with Equation 4, where the stoichiometry is fixed. To resolve this inconsistency, we developed a simple combinatorial model to describe polyA-CA fibre elongation. There is a free energy penalty for bringing an unbound polyA chain

Figure 5: Van 't Hoff plot of TE data at different cyanuric acid concentrations. Coloured symbols represent experimental data from TE traces, solidcoloured lines represent a global fit of Equation 4 and dashed coloured lines represent a global fit of Equation 9. Solid and dashed lines are virtually superimposed on each other. Experimental errors are smaller than the size of the symbols.

in close proximity to the end of a fibre, $\Delta G_{\text {polyA }}=\Delta H_{\text {polyA }}-T \Delta S_{\text {polyA }}$. This is compensated by energetically favourable binding of CA molecules to the newly-created 15 binding sites. All CA molecules are assumed

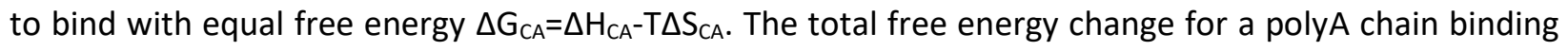
along with a specific configuration of $c$ CA molecules is $\Delta \mathrm{G}_{\text {polyA }}+c \Delta \mathrm{G}_{\mathrm{cA}}$. Overall, the apparent equilibrium dissociation constant for polyA chain binding is given by ${ }^{28}$

$$
\left(K_{e}\right)^{-1}=K_{\text {poly } A}\left(1+K_{C A}[C A]\right)^{15}
$$

where $K_{\text {polyA }}=\exp \left(-\Delta G_{\text {polyA }} / R T\right)$ and $K_{C A}=\exp \left(-\Delta G_{C A} / R T\right)$. The average number of $C A$ molecules can be calculated using the following equation

$$
\langle c\rangle=15 \frac{K_{C A}[C A]}{1+K_{C A}[C A]}
$$

and the fraction of bound states with a given number of CA molecules can be calculated by

$$
\theta_{c}=\left(\frac{15 !}{c !(15-c) !}\right) \frac{K_{C A}[C A]^{c}}{\left(1+K_{C A}[C A]\right)^{15}}
$$

We fit Equation 9 to the TE data, obtaining excellent agreement, and extracting $\Delta \mathrm{H}_{\text {polyA }}, \Delta \mathrm{S}_{\text {polyA }}, \Delta \mathrm{H}_{\mathrm{CA}}$, and $\Delta \mathrm{S}_{\mathrm{CA}}$ (Figure 5, Supplementary Table 3). These parameters allowed us to calculate the fractions of polyA chains with different numbers of CA molecules bound at different temperatures and [CA], providing a highly detailed description of assembly (Figure 6c). Under highly stabilizing conditions of high [CA] and 
low temperature, the Equations predict that almost all binding sites are filled, in agreement with previous dialysis experiments. ${ }^{17}$ Importantly, Equation 9 and Equation 10 explain why we observe 10 bound CA, and not more or less, even though experiments were performed at different [CA]. All experiments used $25 \mu \mathrm{M}$ polyA, which means that we only detected $K_{e}$ values between about $3 \mu \mathrm{M}$ and $22 \mu \mathrm{M}$ in all cases. This implies that the $\mathrm{K}_{\mathrm{CA}}[\mathrm{CA}]$ values are nearly identical in all experiments (since $K_{\text {poly }}$ does not change much with temperature) From Equation 10 , this implies that $\langle c\rangle$ is very similar in all experiments, ranging from 10 to 11, and in excellent agreement with the simple fit described in the previous section.

High valence supramolecular systems have many useful properties that are only just beginning to be explored, such as the ability to self-heal, responsiveness to stimuli, and simple, inexpensive chemical derivatization. Examples include small molecule-directed nucleic acid assembly (CA + polyadenosine or polydeoxyadeonsine ${ }^{14,17}$; melamine + polythymine ${ }^{29}$ ) and non-covalent polymer crosslinking via multiple metal chelation ${ }^{25}, 30$ or host/guest interactions ${ }^{31,32}$. Equation 9 and Equation 10 can serve as starting points for quantitatively describing assembly in such systems, where simple probabilistic considerations ensure that some of the binding sites will remain vacant under many conditions. Furthermore, we find that TEderived data are sufficient to extract the relevant thermodynamic parameters robustly, providing a new avenue for gaining insight into these complex materials.

\section{Generality of the Method}

Our aim for the TE mapping method is that it can be used as a general tool to determine the thermodynamic parameters of supramolecular assembly when standard thermal melting and annealing experiments are unsuitable for thermodynamic analysis. Towards this end, we have also tested the method on a tetrameric intermolecular guanine quadruplex (G4) in aqueous buffer, and zinc-porphyrin self-assembly in mixture of methylcyclohexane and chloroform. In both cases, we obtained series of concave-up and concave-down arcs, similar to those of the polyA-CA fibres (Supplementary Figure 4). In parallel, we used computer simulations to model the TE experiment for different types of self assembling systems and observed two patterns of behaviour: either all the extrema aligned with the equilibrium curve or the maxima for the cooling curves and minima for the heating curves were offset from one another (Supplementary Figure 4). This provides a useful guide for interpreting TE data on new systems of interest: when the extrema align, they can be used to trace out the equilibrium curve (as for polyA-CA fibres and the intermolecular G4). When they are offset, they cannot be directly equated to equilibrium temperature/concentration pairs (as for the zinc porphyrin system), although the data are still information-rich, as detailed in the Supplementary Information. Fortunately, many slowly assembling supramolecular structures are amenable to the TE approach and in these cases it provides thermodynamic information that is not readily available from other sources. For example, a polyA-CA $[\mathrm{M}]_{\mathrm{c}}$ dataset similar to the one reported here would require a scan rate of $<0.001 \mathrm{~K} \mathrm{~min}^{-1}$ in traditional melting measurements, leading to experiments on the impractically long timescale of a month. Our study demonstrates how the ready availability of high-quality thermodynamic dynamic data can lead to new insights, such as the prevalence of unfilled CA binding sites in polyA-CA fibres, and provides an opportunity to test theoretical developments, such as our master equation for high-valence assembly. These advances would not have been realistically possible for polyA-CA structures using previously-existing methods. It is worthwhile noting that quite a few other types of slowly assembling systems have been reported including viral capsids, amyloid fibrils and other supramolecular polymers, ${ }^{10,33,34}$ and that other slowly-equilibrating systems such as spin-crossover ${ }^{35,36}$ and nanoparticle agglomeration ${ }^{37}$ could benefit from the TE mapping approach. 
Furthermore, we may be substantially underestimating the number of potentially interesting slowly assembling systems. Anecdotally, molecules that initially show hysteresis in thermal melts are often disfavoured for further characterization, since adequate tools for thermodynamic analysis have been lacking, leading to underreporting of these types of structures. We believe that the TE mapping method presented here is a big step towards filling this gap in the experimentalist's toolbox. It can be applied to a wide variety of systems using common benchtop laboratory equipment and measurement times are on the order of 10 hours. The experiments are straightforward to set up and a typical analysis (eg van ' $t$ Hoff plot), can be performed entirely using standard spreadsheet software (see Supplementary Information). We believe that the TE mapping method will prove generally useful to the supramolecular chemistry community. 


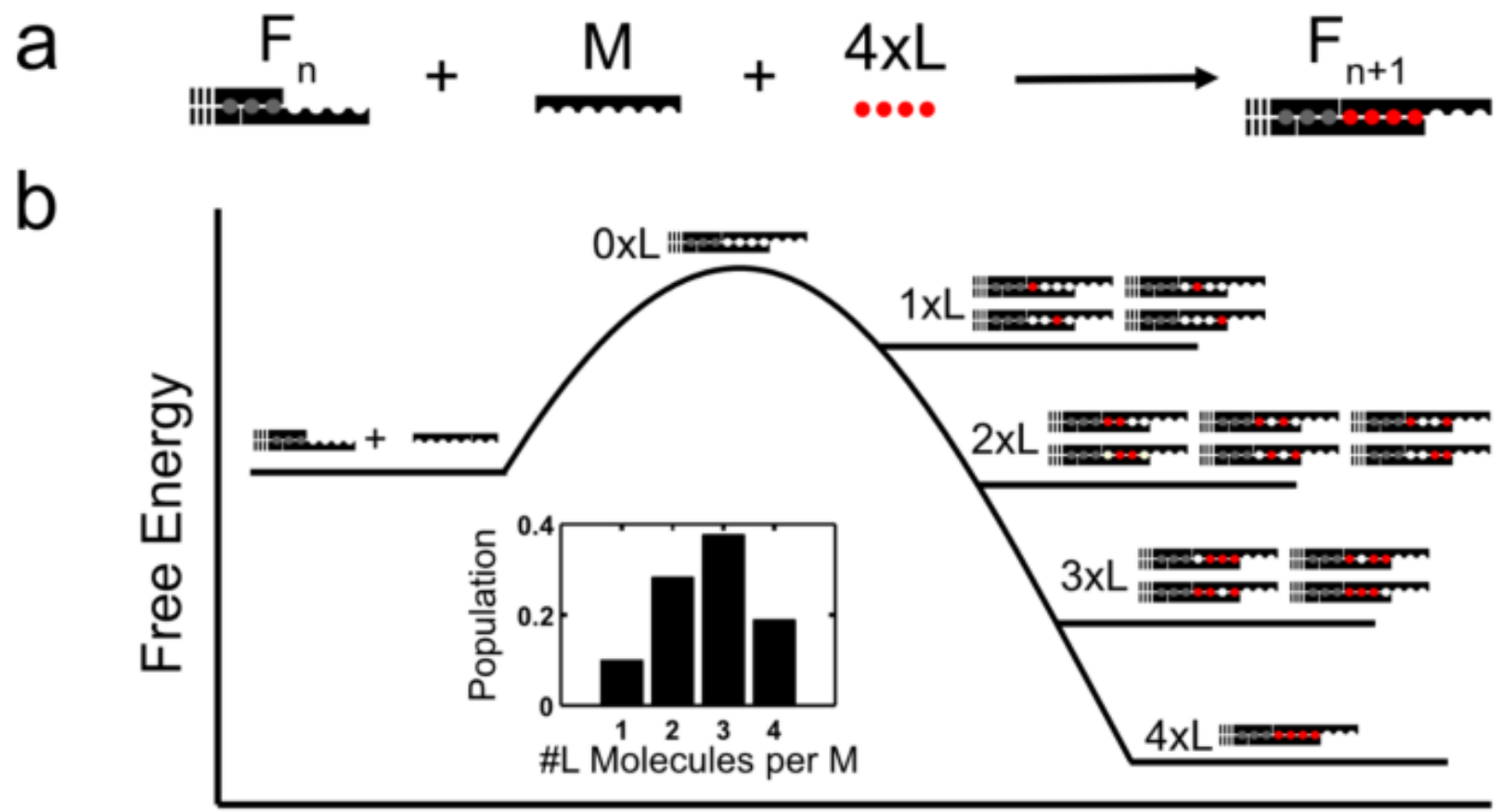

Reaction Coordinate

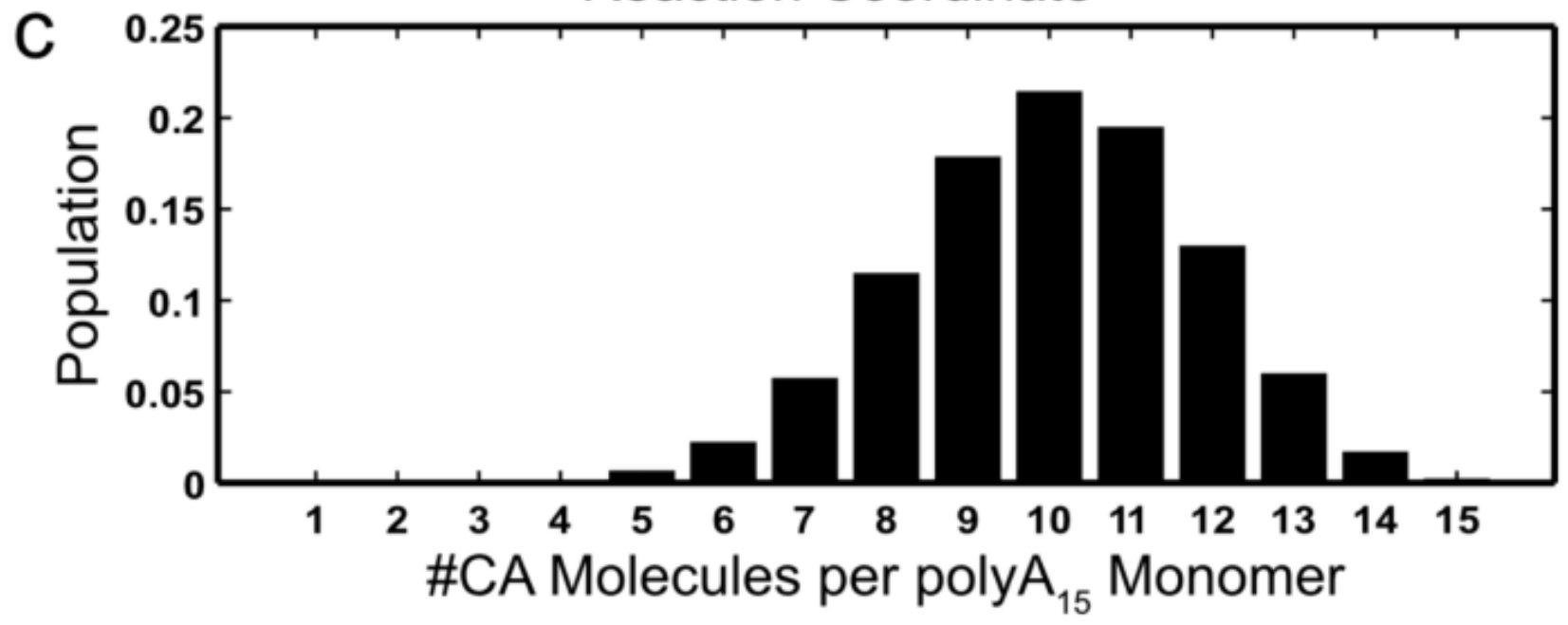

Figure 6: a) A simple constant stoichiometry assembly model where the end of a growing fiber $\left(F_{n}\right)$ assembles with one monomer $M$ and 4 ligand molecules $L$ to create a fiber of length $n+1\left(F_{n+1}\right)$. b) $A$ free energy diagram of a variable stoichiometry assembly model where the end of a growing fiber $\left(F_{n}\right)$ can assembly with a monomer $M$ and any number of ligand molecules $L$ up to a maximum of 4 , the insert represents the populations of each stoichiometry. c) The populations of each stoichiometry for the self assembly of polyA-CA fibers at $25^{\circ} \mathrm{C}$ with a concentration of $12.5 \mathrm{mM}$ CA. 


\section{Online Methods}

\section{Materials}

Cyanuric acid (CA), tris(hydroxymethyl)aminomethane (Tris), magnesium chloride hexahydrate $\left(\mathrm{MgCl}_{2} \cdot 6\right.$ $\mathrm{H} 2 \mathrm{O}$ ), sodium chloride ( $\mathrm{NaCl}$ ), glacial acetic acid and urea were used as purchased from Sigma-Aldrich. Boric acid was obtained from Fisher Scientific and used as supplied. Acrylamide/bis-acrylamide (40\% 19:1) solution, ammonium persulfate and tetramethylethylenediamine (TEMED) were used as purchased from BioShop Canada Inc.

1xTBE (Tris-boric acid-EDTA) buffer was composed of $45 \mathrm{mM}$ Tris, $45 \mathrm{mM}$ boric acid and $2 \mathrm{mM}$ EDTA at $\mathrm{pH}$ 8.3. 1xAcMg buffer was composed of $40 \mathrm{mM}$ acetic acid, $7.6 \mathrm{mM} \mathrm{MgCl} \cdot 6 \mathrm{H} 2 \mathrm{O}$, with $\mathrm{pH}$ adjusted to 4.5. Buffers and samples were prepared with Milli-Q water.

$d\left(A_{15}\right)$ oligonucleotides were synthesized on a Mermade-12 synthesizer, purified by denaturing polyacrylamide gel electrophoresis (PAGE 20\%, 1xTBE running buffer, $8 \mathrm{M}$ urea) and desalted with GelPak desalting columns from Glen Research. Purity of the strand was confirmed by HRMS (Calculated mass: 4635.18; Observed mass: 4634.28).

Stock solutions of $20 \mathrm{mM} \mathrm{CA}$ were prepared by dissolution in $100 \mathrm{~mL}$ of Milli-Q water in a volumetric flask and adjusted with acetic acid to $\mathrm{pH} 4.5$. To properly dissolve and degas the solutions, they were heated at $65{ }^{\circ} \mathrm{C}$ and sonicated, then cooled down to room temperature before being used.

Samples of $100 \mu \mathrm{L}$ of polyA $\mathrm{A}_{15}(25 \mu \mathrm{M})$ and CA $(7.5,10.0,12.5$ and $15.0 \mathrm{mM})$ in pH $4.5 \mathrm{Mg}(\mathrm{OAc})_{2}$ buffer $(7.6$ $\mathrm{mM})$ were made in quadruplicates. A thin layer $(\sim 30 \mu \mathrm{L})$ of silicon oil was applied on top to prevent evaporation during experiments.

\section{Instrumentation}

UV-Vis absorbance-based quantification of $d\left(A_{15}\right)$ was performed on a Nanodrop Lite spectrophotometer from Thermo Scientific. DNA purification by PAGE was carried out on a $20 \times 20 \mathrm{~cm}$ vertical acrylamide Hoefer 600 electrophoresis unit.

UV-Vis absorbance studies were performed using a $1.0 \mathrm{~mm}$ quartz cuvette and monitored at $260 \mathrm{~nm}$ on an Agilent Cary 300 Series UV-Vis Spectrophotometer equipped with a Peltier temperature controller and water recirculator. A variable temperature range which started from $50-40{ }^{\circ} \mathrm{C}$ and went down to $10-4{ }^{\circ} \mathrm{C}$ was scanned at a rate of $0.5^{\circ} \mathrm{C} / \mathrm{min}$ and with a max equilibration time of $30 \mathrm{~min}$. Argon gas and drierite were used to dry the chamber at temperatures below $10^{\circ} \mathrm{C}$.

\section{Data availability}

The MATLAB code for performing the analyses herein is available from the corresponding author upon request.

\section{Code availability}

The data for performing the analyses herein are available from the corresponding author upon request. 


\section{References}

1. Busseron, E.; Ruff, Y.; Moulin, E.; Giuseppone, N., Supramolecular self-assemblies as functional nanomaterials. Nanoscale 2013, 5 (16), 7098-7140.

2. Elemans, J. A.; Rowan, A. E.; Nolte, R. J., Mastering molecular matter. Supramolecular architectures by hierarchical self-assembly. Journal of Materials Chemistry 2003, 13 (11), 2661-2670.

3. Corbett, P. T.; Leclaire, J.; Vial, L.; West, K. R.; Wietor, J.-L.; Sanders, J. K. M.; Otto, S., Dynamic Combinatorial Chemistry. Chemical Reviews 2006, 106 (9), 3652-3711.

4. Medrano, M.; Fuertes, M. A. n.; Valbuena, A.; Carrillo, P. J.; Rodríguez-Huete, A.; Mateu, M. G., Imaging and quantitation of a succession of transient intermediates reveal the reversible selfassembly pathway of a simple icosahedral virus capsid. Journal of the American Chemical Society 2016, 138 (47), 15385-15396.

5. Pinotsi, D.; Buell, A. K.; Galvagnion, C.; Dobson, C. M.; Kaminski Schierle, G. S.; Kaminski, C. F., Direct Observation of Heterogeneous Amyloid Fibril Growth Kinetics via Two-Color Super-Resolution Microscopy. Nano Letters 2014, 14 (1), 339-345.

6. Rennella, E.; Sekhar, A.; Kay, L. E., Self-assembly of human Profilin-1 detected by Carr-PurcellMeiboom-Gill nuclear magnetic resonance (CPMG NMR) spectroscopy. Biochemistry 2017, 56 (5), 692703.

7. Bellot, M.; Bouteiller, L., Thermodynamic description of bis-urea self-assembly: competition between two supramolecular polymers. Langmuir 2008, 24 (24), 14176-14182.

8. Pasternack, R. F.; Goldsmith, J. I.; Szép, S.; Gibbs, E. J., A spectroscopic and thermodynamic study of porphyrin/DNA supramolecular assemblies. Biophysical journal 1998, 75 (2), 1024-1031.

9. Mergny, J.-L.; Lacroix, L., Analysis of thermal melting curves. Oligonucleotides 2003, 13 (6), 515537.

10. Singh, S.; Zlotnick, A. J. J. o. B. C., Observed hysteresis of virus capsid disassembly is implicit in kinetic models of assembly. 2003, 278 (20), 18249-18255.

11. Sambe, L.; de La Rosa, V. R.; Belal, K.; Stoffelbach, F.; Lyskawa, J.; Delattre, F.; Bria, M.; Cooke, G.; Hoogenboom, R.; Woisel, P., Programmable Polymer-Based Supramolecular Temperature Sensor with a Memory Function. Angewandte Chemie International Edition 2014, 53 (20), 5044-5048.

12. Mizuno, K.; Boudko, S. P.; Engel, J.; Bächinger, H. P., Kinetic hysteresis in collagen folding. Biophysical journal 2010, 98 (12), 3004-3014.

13. Dastan, A.; Frith, W. J.; Cleaver, D. J., Thermal Hysteresis and Seeding of Twisted Fibers Formed by Achiral Discotic Particles. The Journal of Physical Chemistry B 2017, 121 (42), 9920-9928.

14. Harkness V, R. W.; Avakyan, N.; Sleiman, H. F.; Mittermaier, A. K., Mapping the energy landscapes of supramolecular assembly by thermal hysteresis. Nature Communications 2018, 9 (1), 3152.

15. Harkness, R. W.; Hennecker, C.; Grün, J. T.; Blümler, A.; Heckel, A.; Schwalbe, H.; Mittermaier, A. K., Parallel reaction pathways accelerate folding of a guanine quadruplex. Nucleic acids research 2021, 49 (3), 1247-1262.

16. Yamaguchi, M., Thermal Hysteresis Involving Reversible Self-Catalytic Reactions. Accounts of Chemical Research 2021, 10226-10234.

17. Avakyan, N.; Greschner, A. A.; Aldaye, F.; Serpell, C. J.; Toader, V.; Petitjean, A.; Sleiman, H. F., Reprogramming the assembly of unmodified DNA with a small molecule. Nature Chemistry 2016, 8, 368.

18. Alenaizan, A.; Fauché, K.; Krishnamurthy, R.; Sherrill, C. D., Noncovalent Helicene Structure between Nucleic Acids and Cyanuric Acid. Chemistry-A European Journal 2021, 27 (12), 4043-4052.

19. Goldstein, R. F.; Stryer, L., Cooperative polymerization reactions. Analytical approximations, numerical examples, and experimental strategy. Biophysical Journal 1986, 50 (4), 583-599. 
20. Arisawa, M.; Iwamoto, R.; Yamaguchi, M., Unstable and Stable Thermal Hysteresis Under Thermal Triangle Waves. ChemistrySelect 2021, 6 (18), 4461-4465.

21. Zhao, D.; Moore, J. S., Nucleation-elongation: a mechanism for cooperative supramolecular polymerization. Organic \& Biomolecular Chemistry 2003, 1 (20), 3471-3491.

22. Markham, N. R.; Zuker, M., DINAMelt web server for nucleic acid melting prediction. Nucleic acids research 2005, 33 (suppl_2), W577-W581.

23. Mikulecky, P. J.; Feig, A. L., Heat capacity changes associated with nucleic acid folding. 2006, 82 (1), 38-58.

24. Self-Processes - Programmed Supramolecular Systems. In Supramolecular Chemistry, 1995; pp 139-197.

25. Hamacek, J. J. M., Self-Assembly Principles of Helicates. Wiley Online Library: 2013; pp 91-124.

26. Paneerselvam, A. P.; Mishra, S. S.; Chand, D. K. J. J. o. C. S., Linear and circular helicates: A brief review. 2018, 130 (7), 96.

27. Hamacek, J.; Borkovec, M.; Piguet, C. J. C. A. E. J., A Simple Thermodynamic Model for Quantitatively Addressing Cooperativity in Multicomponent Self-Assembly Processes-Part 1:

Theoretical Concepts and Application to Monometallic Coordination Complexes and Bimetallic Helicates Possessing Identical Binding Sites. 2005, 11 (18), 5217-5226.

28. Dill, K. A.; Bromberg, S.; Stigter, D., Molecular driving forces: statistical thermodynamics in biology, chemistry, physics, and nanoscience. Garland Science: 2010.

29. Li, Q.; Zhao, J.; Liu, L.; Jonchhe, S.; Rizzuto, F. J.; Mandal, S.; He, H.; Wei, S.; Sleiman, H. F.; Mao, H., A poly (thymine)-melamine duplex for the assembly of DNA nanomaterials. Nature Materials 2020, 19 (9), 1012-1018.

30. Lehn, J.-M.; Rigault, A.; Siegel, J.; Harrowfield, J.; Chevrier, B.; Moras, D., Spontaneous assembly of double-stranded helicates from oligobipyridine ligands and copper (I) cations: structure of an inorganic double helix. Proceedings of the National Academy of Sciences 1987, 84 (9), 2565-2569.

31. Mulder, A.; Huskens, J.; Reinhoudt, D. N., Multivalency in supramolecular chemistry and nanofabrication. Organic \& biomolecular chemistry 2004, 2 (23), 3409-3424.

32. Lin, M.; Dai, Y.; Xia, F.; Zhang, X., Advances in non-covalent crosslinked polymer micelles for biomedical applications. Materials Science and Engineering: C 2020, 111626.

33. Morel, B.; Varela, L.; Conejero-Lara, F., The Thermodynamic Stability of Amyloid Fibrils Studied by Differential Scanning Calorimetry. The Journal of Physical Chemistry B 2010, 114 (11), 4010-4019.

34. Sorrenti, A.; Leira-Iglesias, J.; Markvoort, A. J.; de Greef, T. F.; Hermans, T. M. J. C. S. R., Nonequilibrium supramolecular polymerization. 2017, 46 (18), 5476-5490.

35. Volatron, F.; Catala, L.; Rivière, E.; Gloter, A.; Stéphan, O.; Mallah, T., Spin-Crossover Coordination Nanoparticles. Inorganic Chemistry 2008, 47 (15), 6584-6586.

36. Galán-Mascarós, J. R.; Coronado, E.; Forment-Aliaga, A.; Monrabal-Capilla, M. a.; PinillaCienfuegos, E.; Ceolin, M., Tuning size and thermal hysteresis in bistable spin crossover nanoparticles. Inorganic chemistry 2010, 49 (12), 5706-5714.

37. Kruse, J.; Merkens, S.; Chuvilin, A.; Grzelczak, M., Kinetic and Thermodynamic Hysteresis in Clustering of Gold Nanoparticles: Implications for Nanotransducers and Information Storage in Dynamic Systems. ACS Applied Nano Materials 2020, 3 (9), 9520-9527.

\section{Acknowledgements}

The authors thank Professor E. W. Meijer for supplying the zinc porphyrin sample as well as for the many fruitful discussions of the work. C.D.H. was supported by an NSERC postgraduate scholarship. C.L.B. was 
supported by an NSERC Vanier Canada Graduate Scholarship. H.S. would like to thank NSERC, CIHR, CFI, and the Canada Research Chairs Program for funding. H.S. is a Cottrell Scholar of the Research Corporation. A.M. was supported by NSERC (Discovery grant number 327028-09).

\section{Author Contributions}

H.S. and A.M. conceived the project. C.L.B. acquired all experimental data. C.D.H. performed simulations, data analysis and interpreted the results with A.M. A.M. and H.S. supervised the projects. C.D.H. and A.M. wrote the paper.

\section{Competing Interests}

The Authors declare no competing interests. 